\title{
Deciphering the HIV-host interactome: overcoming the bottleneck of previous approaches
}
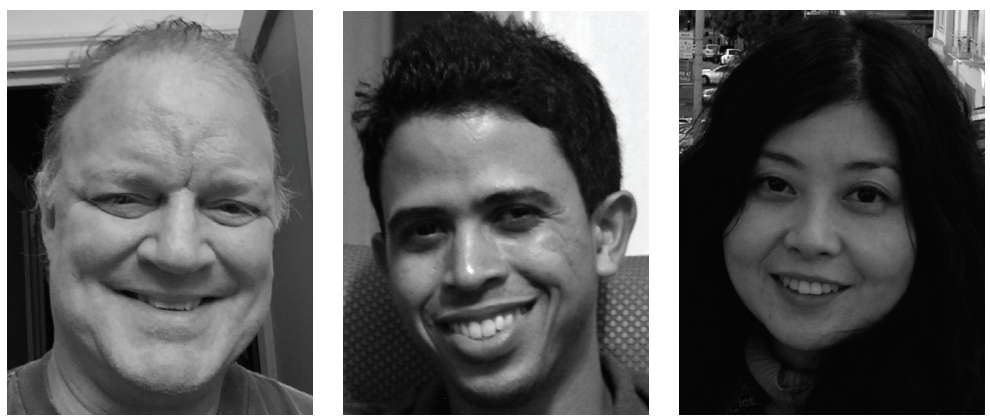

Mark A Muesing ${ }^{* 1,2}$, Kevin D Mohammed ${ }^{1,2,3}$ \& Yang Luo ${ }^{1,2}$

First draft submitted: 31 October 2016; Accepted for publication: 1 November 2016; Published online: 21 December 2016

Given the limited genetic capacity of HIV and the complex molecular events that occur across all phases of infection, it is likely that each viral protein interacts with its own diverse sets of key cellular factors as the virus makes passage through the host cell. In turn, some cellular factors specify antiviral activities that impart defensive measures against further dissemination of the virus within susceptible cell populations. Discovery of these specific virushost protein interactions (the virus-host interactome) may therefore reveal both how the virus gains control of the host and which cellular mechanisms attempt to confound, counteract or subdue the infection. Correspondingly, elucidation of the contacts made between HIV and its requisite host factors during infection would be a great asset not only for the detailed molecular understanding of the 'give and take' of infection, but also, in a more translational sense, the potential to provide many new targets for strategic antiviral intervention. Although RNA interference library screening has previously been used to define those cellular genes having negative impact on HIV replication [1-4], the identity sets of host genes found by this approach are discouragingly incongruent, with few genes shared between different groups of investigators. In addition, these types of screens diminish or abolish [5] host gene expression, removing from experimental consideration those host proteins that, with their reduction or loss, are incompatible with cell viability. Host protein affinity capture is a more direct approach for deciphering the HIV interactome. Unlike RNA interference library screening, it can identify factors with positive, negative or neutral impact on viral replication without perturbation of the intracellular biochemical milieu or cellular growth. The potential power of affinity-tagged approaches was recently demonstrated in a comprehen-

\section{KEYWORDS}

- 3xFLAG epitope - geneticproteomic approach $\cdot$ HIV-host interactome $\cdot$ I-DIRT • replicationcompetent tagged virus $\bullet$ trimeric envelop • Vif

“...elucidation of the contacts made between HIV and its requisite host factors during infection would be a great asset not only for the detailed molecular understanding of the 'give and take' of infection, but also, in a

more translational sense, the potential to provide many new targets for strategic antiviral intervention." 
sive study identifying host binding partners of ectopically overexpressed individual viral proteins [6]. Nonetheless, there are obvious inherent shortcomings using this type of approach as well. In these studies [6], the identification of the viral-host interactome was made piecemeal - in effect, defining the host interactome of a given viral protein as examined in isolation following transient DNA transfection of an individual viral gene. As such, the host interactome for each viral protein was defined without regard to the precise quantitative relationships between the viral protein and its respective host factor(s) that exist during natural infection. Furthermore, and more importantly, these experiments were conducted without the sequential, temporal orchestration of all components of the viral proteome. Without acknowledging this consideration, many host members of the host interactome, recruited by and acting with the virus to affect authentic, sustained infection of target cells, may be missed.

To address this challenge, a new methodology has been developed that overcomes this bottleneck [7]. Using a systematic tagging methodology for the recovery of immunotagged, replicationcompetent viruses, direct probing of virus-host interactions in the context of ensuing, natural viral replication of susceptible lymphocytes has been made possible [7]. This coupled geneticproteomic approach uses random transpositional mutagenesis paired with affinity purification and differential isotopic mass spectrometry to offer a more pertinent and integrated or 'global' version of viral replication, definition of the components of the host interactome acting in concert with the virus as recovered and identified directly from the infection of susceptible target cells. The success of this methodology utilizes saturation linker scanning mutagenesis to introduce a unique endonuclease restriction site at all possible nucleotide positions within a given targeted viral gene segment to pinpoint those rare locations within the virus that can accommodate a small, foreign insertion without loss of sustained cycles of viral replication through susceptible cells in culture. This new site can then serve as a position to accept the specific insertion of a DNA sequence encoding an exogenous epitope tag or other operative elements. In this way, replication-competent, recombinant viruses incorporating a well-characterized peptide tag (i.e., 3xFLAG, which exhibits very high affinity for its cognate antibody) have been recovered [7]
Since the tagged viruses are selected for robust replication competency, isolated without reference to prior structural information, each tagged protein is likely to be engaged with the same repertoire of cellular factors that occur naturally during infection. Because of the limited coding capacity of HIV, we expect that in most cases, the interactions made between the virus and the host cell factor(s) may exist only transiently, the molecular topology provided by the viral protein capable of recruiting different cellular factor(s) at precise times after viral infection, these interactions separated both temporally and spatially over the time course of the viral life cycle. Thus, in conjunction with fast cryofracture cell disruption and immunoaffinity purification [7], we have the ability to capture viral-host interactions and identify both known and novel cellular complexes that directly interact with the viral machinery at specific times over the course of the infectious cycle.

Critical to the success of this strategy, the foreign 3xFLAG tag is stably maintained, inert with respect to many ensuing cycles of spreading viral replication. This allows for the direct affinity capture and identification of viral-host macromolecular complexes by various mass spectrometry methodologies from large-scale amplification of tagged, virally infected cultures. Originally, we selected two contrasting HIV proteins to tag [7]: an abundant, extensively glycosylated structural envelope protein (Env) and a relatively scarce cytosolic accessory factor (Vif) with a critical role in counteracting host-mediated intrinsic immunity against the virus in vivo. Differential isotopic labeling (the I-DIRT technique: differentiation of interactions as random or targeted) was used to assign specific viral-host interactions without bias [8]. I-DIRT is capable of discrimination between specific and nonspecific proteins obtained from isolated protein complexes providing the investigator the ability to assign authentic interactions with unprecedented confidence [8]. For example, comparative analysis between an infected culture of a 3xFLAG-tagged virus and that of the untagged, wild-type parental virus provides high-certainty identification of interactors made by their direct access during cycling infection $[7,8]$. In practice, similarly grown infectious cultures of the $3 \times$ FLAG-tagged virus (labeled in $\mathrm{C}^{12}$ media) and the untagged, wild-type virus (labeled in $\mathrm{C}^{13}$ media) are individually expanded, cell pellets flash frozen in liquid nitrogen, the 
pellets mixed together in equal amounts before cryogenic cellular disruption and magnetic beadmediated, anti-FLAG monoclonal antibody immunoprecipitation and mass spectrometric identification of the constituent host interactor proteins. The discrimination afforded by the I-DIRT technique can be corroborated by the inclusion of an additional I-DIRT experiment in which the natural carbon $\left(\mathrm{C}^{12}\right)$ and isotopically marked carbon $\left(\mathrm{C}^{13}\right)$ are switched, the tagged and untagged comparator HIV cultures grown in the reverse carbon isotope.

Advances in mass spectrometry based proteomic methodologies have revolutionized our ability to decipher the HIV-host interactome (reviewed [9]), allowing the discovery of previously unexplored processes and pathways, providing insights into how HIV commandeers the host machinery and evades the human immune system to persist throughout the lifetime of the infected individual. Toward this goal, by probing virus-host interactions directly in their natural context, several host factors of functional relevance previously obscured from scientific investigation have been identified with high confidence [7]. These host factors impact not only viral propagation in vitro, but also may be critical to the survival of the virus against strong immunological attack by the host in vivo. Since our approach allows for the detection of even transient protein associations made between viral components and its cellular factors [7], we believe the next generation of proteomic methodologies will expand our ability to provide a more dynamic picture of the temporal and spatial relationships made between the virus and its host at defined points within the viral life cycle. Ultimately, such a comprehensive view of the molecular intersections made between HIV and its host will afford the discovery of new cellular interactors, providing the impetus for unique strategies of antiviral intervention against those factors, in part, dispensable in the host but required for viral pathogenesis - some of which may be more durable targets for antiviral intervention and relatively refractory to the emergence of viral resistance. Finally, our approach is broadly applicable to elucidating a large spectrum of pathogen-host interactomes, an understanding the pathophysiological consequences of these specific associations likely to provide strategic targets for antimicrobial intervention.

\section{Financial \& competing interests disclosure}

This work was supported by grants from the National Institutes of Health: R01AI047054, R21AI065321, R01AI081615 $221 A 1097233$ (all to MA Meusing). The authors have no other relevant affiliations or financial involvement with any organization or entity with a financial interest in or financial conflict with the subject matter or materials discussed in the manuscript apart from those disclosed.

No writing assistance was utilized in the production of this manuscript.

\section{References}

1 Brass AL, Dykxhoorn DM, Benita Y et al. Identification of host proteins required for HIV infection through a functional genomic screen. Science 319(5865), 921-926 (2008).

2 Bushman FD, Malani N, Fernandes J et al. Host cell factors in HIV replication: meta-analysis of genome-wide studies. PLoS Pathog. 5(5), e1000437 (2009).

3 Konig R, Zhou Y, Elleder D et al. Global analysis of host-pathogen interactions that regulate early-stage HIV-1 replication. Cell 135(1), 49-60 (2008).
Zhou H, Xu M, Huang Q et al. Genome-scale RNAi screen for host factors required for HIV replication. Cell Host Microbe 4(5), 495-504 (2008).

5 Hultquist JF, Schumann K, Woo JM et al. A Cas9 ribonucleoprotein platform for functional genetic studies of HIV-host interactions in primary human T cells. Cell Rep. 17(5), 1438-1452 (2016).

6 Jager S, Cimermancic P, Gulbahce $\mathrm{N}$ et al. Global landscape of HIV-human protein complexes. Nature 481(7381), 365-370 (2012).
7 Luo Y, Jacobs EY, Greco TM et al. HIV-host interactome revealed directly from infected cells. Nat. Microbiol. 1(7), 16068 (2016).

8 Tackett AJ, Degrasse JA, Sekedat MD, Oeffinger M, Rout MP, Chait BT. I-DIRT, a general method for distinguishing between specific and nonspecific protein interactions. J. Proteome Res. 4(5), 1752-1756 (2005).

9 Luo Y, Muesing MA. Mass spectrometrybased proteomic approaches for discovery of HIV-host interactions. Future Virol. 9(11), 979-992 (2014). 